\title{
A INFLUÊNCIA DA RESPONSABILIDADE SOCIAL CORPORATIVA NA SATISFAÇÃO DOS EMPREGADOS: UM ESTUDO COM MODELAGEM DE EQUAÇÕES ESTRUTURAIS BASEADO EM MÍNIMOS QUADRADOS PARCIAIS
}

Versão do autor aceita publicada online: 18 nov. 2021

Publicado online: 23 nov. 2021

Como citar esse artigo - American Psychological Association (APA): Ceciliano, P. H.,

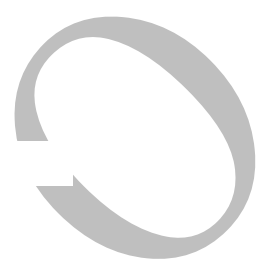

Vieira, P. R. da C., \& Silva, A. C. M. (2021). A influência da responsabilidade social corporativa na satisfação dos empregados: um estudo com modelagem de equações estruturais baseado em mínimos quadrados parciais. Exacta. DOI: https://doi.org/10.5585/exactaep.2021.19002.

\section{Paulo Henrique Ceciliano}

pauloceciliano1980@gmail.com

https://orcid.org/0000-0002-4758-4299

Universidade Estácio de Sá

Mestre em Administração e Desenvolvimento Empresarial pela Universidade Estácio de Sá (MADE/UNESA)

http://lattes.cnpq.br/2266446636153501

\section{Paulo Roberto da Costa Vieira}

https://orcid.org/0000-0003-3742-1127

Doutor em Administração (COPPEAD/UFRJ)

http://lattes.cnpq.br/0164329912899593

\section{Antonio Carlos Magalhães da Silva}

https://orcid.org/0000-0003-4497-3130

Universidade Estácio de Sá

Doutor em Administração (COPPEAD/UFRJ)

http://lattes.cnpq.br/0681931788963814

Professor do Mestrado em Administração e Desenvolvimento Empresarial da Universidade Estácio de Sá (MADE/UNESA)

Bolsista do Programa de Pesquisa e Produtividade da Universidade Estácio de Sá (MADE/UNESA)

Bolsista do Programa de Extensão da Universidade Estácio de Sá (MADE/UNESA)

Professor da Universidade Federal Fluminense (UFF)

\section{RESUMO}

A responsabilidade social corporativa (RSC) desempenha papel crucial nas corporações, sendo entendida como variável estratégica porque, se gerenciada adequadamente, concorrerá para o aumento de participação no mercado. O presente estudo teve como objetivo avaliar a influência que a RSC e a identidade corporativa exercem sobre a satisfação dos empregados. Para tanto, foi realizada pesquisa de natureza quantitativa, com utilização de questionário estruturado e autoadministrado, aplicado à amostra constituída por 293 empregados de uma indústria brasileira do setor de óleo \& 
gás. O tratamento de dados ocorreu por meio da técnica estatística de modelagem de equações estruturais (MEE), baseada em mínimos quadrados parciais (MQP). Os resultados confirmaram que a satisfação dos empregados é influenciada de forma direta e positiva pela RSC e identidade corporativa. A contribuição dos resultados possibilita aos gestores, o alcance de vantagens competitivas no mercado, com investimento em capital humano, o qual constitui recurso intangível, raro e único.

Palavras-chave: Responsabilidade social corporativa; Identidade corporativa; Satisfação dos empregados; Modelagem de equações estruturais.

\section{ABSTRACT}

Corporate social responsibility (CSR) plays an essential role in corporations being understood as a strategic variable, because, if managed properly, it will contribute to increase corporation's market share. This study aimed to assess the influence that CSR and corporate identity have on employee satisfaction. Therefore, a quantitative research was carried out, using a structured and self-administered questionnaire, applied to the sample consisting of 293 employees of Brazilian oil and gas industry. Data processing took place using the structural equation modeling (SEM) statistical technique, based on partial least squares (PLS). The results confirmed that the satisfaction of the employees of the studied corporation is directly and positively influenced by CSR and corporate identity. The contribution of the results makes it possible for corporate managers to achieve competitive advantages in the market by means of investment in human capital which constitutes rare and unique intangible resource.

Keywords: Corporate social responsibility; Corporative identity; Employee satisfaction; Structural equation modeling.

\section{INTRODUÇÃO}

A responsabilidade social corporativa (RSC) é fator de interesse crucial na abordagem da economia sustentável. As corporações cada vez mais investem esforços e a tratam como ativo estratégico valioso. Há estudos que destacam o benefício obtido por corporações com o engajamento em atividades de RSC, tais como a construção de imagem corporativa positiva e conquista de reputação sólida a longo prazo, despertando, simultaneamente, a consciência de seus clientes para o consumo socialmente responsável (Lee \& Lee, 2018; Lin-Hi \& Blumberg, 2018; Ramesh, Saha, Goswami, \& Dahiya, 2019). No entanto, considerando o desenvolvimento de ações de RSC voltadas principalmente para determinados grupos de partes interessadas, percebe-se a escassez de conhecimento acerca dos impactos da RSC sobre o grupo crítico dos empregados (Brieger, Anderer, Fröhlich, Bäro, \& Meynhardt, 2020). 
A RSC exerce importância crucial no atendimento dos objetivos da Comissão Mundial sobre Meio Ambiente e Desenvolvimento, criada pela Organização das Nações Unidas (ONU). No encontro dessa comissão, em 1987, foram determinados os conceitos de Desenvolvimento Sustentável (DS) corporativo (Brundtland, 1987). O DS relativo aos negócios é modelo que busca satisfazer as necessidades dos stakeholders da geração atual, sem comprometer a capacidade de atendimento dos stakeholders das gerações futuras (Pinelli \& Maiolini, 2017). Com o incentivo das práticas de RSC, as corporações respeitam cada vez mais as relações sociais dos seus empregados e demais stakeholders. Portanto, as iniciativas de RSC maximizam o atendimento das necessidades do capital humano das corporações. O capital humano é componente da dimensão social da sustentabilidade corporativa e está relacionado ao respeito aos direitos humanos, à capacidade de melhorar habilidades, motivação e lealdade de empregados e parceiros (Allameh, 2018). No presente trabalho, são estudadas as relações do comportamento responsável corporativo e seus efeitos sobre o capital humano.

As corporações que se empenham em atividades de RSC têm maior probabilidade de provocar emoções positivas nos seus empregados, como orgulho e prestígio. Os indivíduos sentem que participam de corporação que contribui para o bemestar da sociedade. Este fator promove a satisfação dos empregados (Singhapakdi, Lee, Sirgy, Roh, Senasu, \& Grace, 2019). Os valores éticos responsáveis corporativos conquistam a identificação e concordância dos empregados, ou seja, os empregados se mantêm dispostos a direcionar seu comportamento para atividades alinhadas aos objetivos e valores da corporação para as quais trabalham (Onkila, 2015).

Os valores organizacionais alinhados com o bem-estar geral da sociedade têm relação direta com o aumento do comprometimento e com a satisfação dos empregados das corporações (Zhu, Yin, Liu, \& Lai, 2014). Outra relação que precisa ser estudada é a influência do comportamento dos empregados sobre os resultados corporativos, ou seja, como age o mecanismo de identificação corporativa em meio a iniciativas responsáveis das corporações (Balmer, 2017). O prestígio externo percebido pelos empregados nas corporações é definido como identidade corporativa (Dutton \& Dukerich, 1991). No que tange às consequências da identidade corporativa nas corporações, quanto mais positiva for a percepção do empregado de que sua corporação desfruta de prestígio externo, maior serão tanto a sua identificação com a organização quanto o seu nível de comprometimento com a corporação (Bartels, Pruyn, De Jong, \& Joustra, 2007). 
Compreender o impacto da RSC nos empregados é relevante para o desempenho corporativo, pois tal variável aumenta o compromisso organizacional, aprimora a percepção de cidadania corporativa e foca na produtividade no trabalho (El-Kassar, Yunis, \& El-Khalil, 2017). Compartilhando essa mútua colaboração, o capital humano dentro das corporações torna-se elemento de fundamental importância para os seus vários stakeholders, grupos ou indivíduos que se beneficiam ou são prejudicados pela ação corporativa, incluindo seus próprios direitos, que podem ser violados ou respeitados (Odriozola \& Baraibar-Diez, 2017). O desempenho favorável dos empregados, com seu apoio e confiança, convergem diretamente aos objetivos almejados pelas corporações (Chang, 2015).

Na América Latina, têm-se desenvolvido diversas iniciativas de RSC, alinhando ações das corporações em prol do DS nos setores que atuam. O presente estudo se concentra na lacuna teórica a cerca dos valores organizacionais voltados para o bem estar geral da sociedade, especificamente na relação direta com o aumento do comprometimento e satisfação dos empregados nas corporações (Singhapakdi et al., 2019; Zhu, Yin, Liu, \& Lai, 2014). Em suma, o objetivo do presente estudo é avaliar a influência direta da RSC e da identidade corporativa sobre a satisfação dos empregados, colaborando para o desempenho do DS corporativo a longo prazo. O estudo de caso foi aplicado numa corporação brasileira de Oleo \& gas.

\section{REVISÃO DE LITERATURA}

\subsection{RESPONSABILIDADE SOCIAL CORPORATIVA}

O conceito de RSC é composto da integralização voluntária das questões sociais e ambientais nas atividades comerciais corporativas e nas relações com os stakeholders.

A RSC é frequentemente entendida como ações que vão além do que é prescrito em lei. Em seu sentido mais amplo, a RSC enseja preocupação com as necessidades e objetivos da sociedade, a qual vai além do meramente econômico (Eells \& Walton, 1969). As corporações devem considerar a responsabilidade ambiental, social e econômica na sua tomada de decisões, indo além da pura filantropia (Turker, 2009).

O primeiro estudo que estabeleceu o conceito de RSC foi apresentado por Bowen (1953, p. 6), considerando que é obrigação dos empreendedores "perseguir políticas, tomar decisões ou seguir ações desejáveis de acordo com os objetivos e valores da nossa sociedade". O conceito de RSC amplamente aceito é o proposto por Carroll (1979), 
modelo de base piramidal com abrangência nas quatro dimensões da RSC: econômica, jurídica, ética e discricionária. A ideia básica da RSC nesse modelo é que os negócios e a sociedade se entrelaçam em vez de formarem entidades distintas.

A literatura sobre RSC está em desenvolvimento; do ponto de vista organizacional, as demandas de DS implicam iniciativas de RSC. No nível microeconômico, as corporações estão agregando a visão de DS em suas estratégias, tornando-as fonte de vantagem competitiva, a tal ponto que nenhuma grande corporação pode se dar ao luxo de não possuir medidas voltadas à RSC (McCarthý, 2018). Nesse novo contexto, a noção de stakeholders é central: as corporações devem prestar atenção às expectativas das partes interessadas em seu desenvolvimento (Odriozola \& BaraibarDiez, 2017). A RSC está evoluindo para esta nova orientação voltada ao DS nas estratégias das corporações, com a finalidade de alcançar a Sustentabilidade Corporativa (Pinelli \& Maiolini 2017).

Para alcançar o patamar de sustentalidade corporativa, deve-se obter a projeção de imagem socialmente responsável. Isto é facilitado pela divulgação de informações quanto à governança corporativa, a qual, em inglês, é representada pela expressão environmental, social and corporate governance (ESG), estabelecendo relação direta entre o desempenho da gestão ambiental, social e de governança com a rentabilidade das empresas a longo prazo (Hoang, 2018). O relatório de informações ESG fornece perspectiva abrangente sobre o desempenho e o valor da corporação por várias perpectivas distintas, solidificando a reputação corporativa por meio das iniciativas de RSC (Odriozola \& Baraibar-Diez, 2017).

A utilização de normas também é fundamental para o estabelecimento das diretrizes da RSC nas corporações. A norma internacional SA 8000 exerce cobertura dos trabalhadores sobre questões de direitos humanos (Murmura, Bravi, \& Palazzi, 2017). A norma ISO 26000 promove o desenvolvimento do capital humano nas corporações, justamente por suas características voltadas para os empregados no ambiente de trabalho.

Ao contrário da literatura econômica tradicional, as contribuições de RSC consideram o capital humano como o fator produtivo mais nobre, porque é o único que têm capacidade de criar e inovar (Barakat, Isabella, Boaventura, \& Mazzon, 2016). A motivação do capital humano possibilita não apenas aumento de produtividade, mas também o desenvolvimento de novos produtos e processos. Inovação é o que diferencia as corporações e a própria dinâmica de mercado (Schumpeter, 1984). A visão da inovação permite a ampliação do market share corporativo. 


\subsection{IDENTIDADE CORPORATIVA}

A identidade corporativa, também reconhecida na literatura acadêmica como prestígio externo percebido ou imagem externa interpretada, trata da opinião que os empregados têm sobre como a corporação é percebida pelos stakeholders externos (Abratt, \& Mingione, 2017). Do mesmo modo, é resultante de diversas fontes de informação, incluindo opiniões de grupos de referência, comunicação interna, boca a boca, publicidade e informações externas controladas pela corporação. Desse modo, os empregados têm percepções sobre como as pessoas de fora veem sua corporação (Lee, 2020; Podnar \& Golob, 2017).

Vários estudos observaram consequências positivas da identidade corporativa (Abratt, \& Mingione, 2017; Bartels, Pruyn, De Jong, \& Joustra, 2007; Boğan \& Dedeoğlu, 2020). Quanto maior a percepção dos empregados que sua corporação é prestigiada externamente, mais provavelmente se identificam com a corporação e apresentam comprometimento elevado ou apego afetivo (Smidts, Pruyn, \& Van Riel, 2001).

Quanto mais concretas as ações de bem-estar com os empregados, mais eles acreditam que a corporação está disposta a ceder favores aos seus membros internos sem esperar algo em troca. Estas ações estão além da simples troca de trabalho por remuneração. As ações de bem-estar e cuidado com o capital humano desenvolvido pelos empregados nas corporações incluem assistência médica, segurança no trabalho, política de emprego de qualidade, assistência à educação, igualdade de oportunidades para todos, rejeição do trabalho infantil, respeito aos direitos humanos, políticas de não discriminação no local de trabalho, equilíbrio da vida pessoal e profissional e treinamento vocacional (Haski-Leventhal, Roza, \& Meijs, 2017).

Percepções de justiça no local de trabalho afetam o bem-estar dos empregados; o ambiente justo e ético possibilita classificar a corporação como um bom lugar para trabalhar. Imparcialidade em relação às atitudes da alta gerência, tanto em termos gerais quanto em responsabilidade social, afetam negativamente a motivação e o comprometimento dos empregados (Aguilera, Rupp, Williams, \& Ganapathi, 2007). As iniciativas de RSC direcionadas aos stakeholders externos são percebidas pelos empregados como aspectos especiais da justiça corporativa, principalmente as ações de proteção ambiental e o investimento em comunidades locais. Essas iniciativas 
influenciam positivamente a atitude e comportamento dos empregados em relação à corporação. Ações de RSC percebidas pelos empregados como filantropia e voluntariado influenciam positivamente as intenções de construir relacionamentos comuns com sua corporação, ou seja, aumenta a vontade comunitária (Hon \& Grunig, 1999; Lee, 2020).

O efeito positivo de prestígio e orgulho corporativo, sobre a percepção dos empregados, conduz ao aumento do comprometimento, lealdade e satisfação no trabalho (Bravo, Matute, \& Pina, 2017).

\subsection{SATISFAÇÃO DOS EMPREGADOS}

A definição de satisfação no trabalho mais utilizada na pesquisa acadêmica foi elaborada por Locke (1976, p. 1304), que determina satisfação no trabalho como "estado emocional positivo ou prazeroso resultante da avaliação do trabalho ou das experiências profissionais". Compreender a satisfação no trabalho é crucial no contexto do comportamento corporativo. Pesquisas anteriores revelaram que a satisfação no trabalho é importante porque influencia o desempenho e o comprometimento no trabalho, a satisfação do cliente, a retenção no emprego e o absentismo do empregado (Ashton, 2018; Book, Gatling, \& Kim, 2019; Yousef, 2017).

A satisfação no trabalho pode ser avaliada de forma positiva ou negativa. Tal julgamento pode ser feito sobre a atividade realizada no trabalho ou sobre a situação profissional (Jung \& Yoon, 2015). As emoções estão constantemente ligadas a essas avaliações; por isso, a satisfação no trabalho envolve tanto a emoção quanto a atitude de algum indivíduo em relação à avaliação de seu trabalho (Kampkötter, 2017). Os empregados, durante sua vida laboral, empreendem tempo e energia no local de trabalho. Por esse motivo, é essencial que as corporações concedam garantias de que as necessidades dos empregados serão atendidas, com recursos e condições cedidos pela corporação. 0 bem-estar dos empregados está direta ou indiretamente associado ao seu desempenho no trabalho (Yousef, 2017).

As ações corporativas em benefício dos stakeholders podem levar a emoções positivas entre os empregados, como orgulho, entusiasmo, gratidão e satisfação (Book, Gatling, \& Kim, 2019; Judge, Weiss, Kammeyer-Mueller, \& Hulin, 2017). De outra forma, as ações que têm impacto negativo sobre os stakeholders podem levar a emoções negativas para os empregados, incluindo constrangimento, raiva e medo (El Akremi, Gond, Swaen, De Roeck, \& Igalens, 2018). A RSC torna-se importante estratégia para o 


\begin{tabular}{|c|c|c|c|}
\hline Variável latente & Variável observada & $\begin{array}{c}\text { Autores } \\
\text { relevantes }\end{array}$ & Definições \\
\hline & $\begin{array}{l}\text { Rscorp_4- } \\
\text { políticas de não } \\
\text { discriminação na } \\
\text { remuneração e } \\
\text { promoção dos } \\
\text { empregados } \\
\end{array}$ & $\begin{array}{l}\text { Dhanesh, 2014, } \\
\text { p.144 }\end{array}$ & $\begin{array}{l}\text { Item da dimensão legal de RSC, os } \\
\text { resultados deste estudo mostraram que a } \\
\text { dimensão legal de RSC se relacionou de } \\
\text { forma mais significativa com o } \\
\text { compromisso e com a satisfação dos } \\
\text { empregados. }\end{array}$ \\
\hline & $\begin{array}{l}\text { Rscorp_5 - } \\
\text { oferta de ajuda aos } \\
\text { empregados }\end{array}$ & $\begin{array}{l}\text { El-Kassar et } \\
\text { al., 2017, p. } 12\end{array}$ & $\begin{array}{l}\text { O comportamento dos funcionários no } \\
\text { sentido de apoiar os outros funcionários, } \\
\text { alinhado as estratégias e objetivos da } \\
\text { organização. Indica um ambiente de } \\
\text { trabalho saudável e uma adequação que } \\
\text { serve à missão da organização, apoiando } \\
\text { sua busca por vantagem competitiva. }\end{array}$ \\
\hline & $\begin{array}{l}\text { Rscorp_6 - } \\
\text { bem-estar da } \\
\text { comunidade }\end{array}$ & $\begin{array}{l}\text { Aguilera et al., } \\
\text { 2007, p. } 836\end{array}$ & $\begin{array}{l}\text { Desenvolveram um modelo teórico } \\
\text { multinível para explorar como as empresas } \\
\text { desenvolvem mudança social positiva ao } \\
\text { se envolver em iniciativas de RSC. }\end{array}$ \\
\hline & $\begin{array}{l}\text { Rscorp_7 } 7- \\
\text { atitude benéfica em } \\
\text { favor de pessoas } \\
\text { vulneráveis }\end{array}$ & $\begin{array}{l}\text { Ramesh et al., } \\
2019, \text { p. } 377 \text { e } \\
\text { p. } 378\end{array}$ & $\begin{array}{l}\text { A maioria das empresas com interesse no } \\
\text { bem-estar público compromete-se com } \\
\text { atividades que tragam mudanças para a } \\
\text { sociedade carente. Exercendo } \\
\text { desenvolvimento em iniciativas de } \\
\text { educação infantil, desenvolvimento de } \\
\text { habilidades, saúde, desenvolvimento rural } \\
\text { e sustentabilidade ambiental. }\end{array}$ \\
\hline & $\begin{array}{l}\text { Rscorp_8 - } \\
\text { conquista sucesso sem } \\
\text { pisar em outras } \\
\text { pessoas }\end{array}$ & $\begin{array}{l}\text { Hon \& Grunig, } \\
1999 \text {, p.8 }\end{array}$ & $\begin{array}{l}\text { Desenvolveram escalas para as dimensões } \\
\text { de confiança, mutualidade (cuidado com o } \\
\text { próximo), comprometimento e satisfação. } \\
\text { Incluindo percepções de integridade e } \\
\text { competência. }\end{array}$ \\
\hline & $\begin{array}{l}\text { Idcorp_1- } \\
\text { percepção de local de } \\
\text { prestígio para } \\
\text { trabalhar }\end{array}$ & $\begin{array}{l}\text { Smidts, Pruyn, } \\
\& \text { Van Riel, } \\
\text { 2001, p. } 1052\end{array}$ & $\begin{array}{l}\text { Observaram que os indivíduos se } \\
\text { identificam com um grupo para aumentar } \\
\text { sua auto-estima: quanto mais prestigiosa } \\
\text { alguém percebe que sua organização é, } \\
\text { maior o potencial de aumento da auto- } \\
\text { estima por meio da identidade corporativa. }\end{array}$ \\
\hline & $\begin{array}{l}\text { Idcorp_2- } \\
\text { boa percepção da } \\
\text { comunidade }\end{array}$ & $\begin{array}{l}\text { Abratt \& } \\
\text { Mingione, } \\
2017, \text { p.3 }\end{array}$ & $\begin{array}{l}\text { A identidade corporativa é uma " entidade } \\
\text { viva " que uma vez lançada e aceita, não } \\
\text { pertence mais à gestão de uma } \\
\text { organização, mas a todas as suas partes } \\
\text { interessadas: clientes, funcionários, } \\
\text { acionistas, empresas e fornecedores. }\end{array}$ \\
\hline & $\begin{array}{l}\text { Idcorp_3 - } \\
\text { boa percepção de } \\
\text { empregados de outras } \\
\text { corporações }\end{array}$ & $\begin{array}{l}\text { Dutton \& } \\
\text { Dukerich, 1991, } \\
\text { p.549 }\end{array}$ & $\begin{array}{l}\text { Identificaram que mudanças na identidade } \\
\text { corporativa impulsionam a motivação do } \\
\text { investimento pessoal, voltado as } \\
\text { preocupações dos membros da } \\
\text { organização, em como a imagem } \\
\text { corporativa interna afeta a visão que os } \\
\text { outros tinham de si mesmos. }\end{array}$ \\
\hline \multirow{2}{*}{$\begin{array}{l}\text { Satisfação dos } \\
\text { empregados } \\
\text { (SATISF) }\end{array}$} & $\begin{array}{l}\text { Satisf_1 - } \\
\text { natureza do trabalho }\end{array}$ & \multirow{2}{*}{$\begin{array}{l}\text { Siqueira, 2008, } \\
\text { p.261 }\end{array}$} & $\begin{array}{l}\text { Contentamento com o interesse despertado } \\
\text { pelas tarefas, com a capacidade de } \\
\text { absorção do trabalhador e com a variedade } \\
\text { das tarefas. }\end{array}$ \\
\hline & $\begin{array}{l}\text { Satisf_2- } \\
\text { qualidade da liderança }\end{array}$ & & $\begin{array}{l}\text { Contentamento com a organização e } \\
\text { capacidade profissional do chefe, com o } \\
\text { seu interesse pelo trabalho dos } \\
\text { subordinados e entendimento entre eles. }\end{array}$ \\
\hline
\end{tabular}




\begin{tabular}{|c|c|c|c|}
\hline Variável latente & Variável observada & $\begin{array}{c}\text { Autores } \\
\text { relevantes }\end{array}$ & Definições \\
\hline & $\begin{array}{l}\text { Satisf_3- } \\
\text { promoções no } \\
\text { trabalho }\end{array}$ & & $\begin{array}{l}\text { Contentamento com o número de vezes } \\
\text { que já recebeu promoções, com as } \\
\text { garantias oferecidas a quem é promovido, } \\
\text { com a maneira da empresa realizar } \\
\text { promoções e com o tempo de espera pela } \\
\text { promoção. }\end{array}$ \\
\hline & $\begin{array}{l}\text { Satisf_4 - } \\
\text { estrutura salarial }\end{array}$ & & $\begin{array}{l}\text { Contentamento com o que o trabalhador } \\
\text { recebe como salário, comparando com o } \\
\text { quanto o indivíduo efetivamente trabalha, } \\
\text { com sua capacidade profissional, com o } \\
\text { custo de vida e com os esforços executados } \\
\text { na realização do trabalho. }\end{array}$ \\
\hline & $\begin{array}{l}\text { Satisf_5 - } \\
\text { grupo de trabalho }\end{array}$ & & $\begin{array}{l}\text { Contentamento com a colaboração, a } \\
\text { amizade, a confiança e o relacionamento } \\
\text { mantido com os colegas de trabalho. }\end{array}$ \\
\hline
\end{tabular}

Fonte: Elaboração própria.

\subsection{DESENVOLVIMENTO DAS HIPÓTESES}

A RSC implica em tratar o capital humano como fator raro e de difícil substituição, desenvolvendo ações práticas que motivam o trabalhador, tal como garantir condições ótimas no ambiente de trabalho, o que influencia a satisfação do trabalhador. As corporações com altos níveis de engajamento nas práticas de RSC têm maior probabilidade de atrair empregados altamente qualificados, evitar altas taxas de rotatividade e promover o comprometimento e a motivação dos empregados (Khaskheli, Jiang, Raza, Qureshi, Khan, \& Salam, 2020). Satisfação elevada e comprometimento do capital humano proporcionam desempenho corporativo superior e atraem vantagens competitivas de forma estratégica (Harter, Schmidt, \& Hayes, 2002). Esses argumentos convergem para a seguinte hipótese:

\section{Hipótese 1 (H1): A RSC impacta diretamente na satisfação do empregado.}

A RSC tornou-se construto essencial para obter efeito positivo na identidade corporativa, especificamente na percepção que os empregados têm da corporação. Tentativas de melhorar as condições de trabalho são expressas no desenvolvimento de códigos de conduta corporativa e na adoção de padrões globais que preenchem a função de estrutura para fazer cumprir as leis nacionais (Caldwell, \& Peters, 2018). Quando os empregados percebem que sua corporação está buscando se manter responsável perante a sociedade, ajudando a comunidade, doando recursos ou fornecendo esforços 
voluntariamente, é mais provável que acreditem que outras pessoas avaliem sua corporação positivamente (Hon \& Grunig, 1999).

Essa visão tem base no pressuposto de que os participantes de práticas de RSC são submetidos ao processo de identificação. Eles experimentarão moral mais elevado, autoestima e compartilharão valores com sua organização por meio de sua participação e trabalho (Singhapakdi et al., 2019).

Dessa forma, conclui-se que as corporações mais comprometidas com os princípios de RSC tenham maior probabilidade de evocar avaliações positivas dos empregados a respeito de seu próprio trabalho. De forma similar, atitudes negligentes em relação aos princípios de RSC podem criar avaliações negativas dos empregados. Tais argumentos possibilitam a seguinte hipótese:

\section{Hipótese 2 (H2): A RSC impacta diretamente na identidade corporativa.}

A satisfação no trabalho está fortemente relacionada a políticas e procedimentos realizados pela corporação para gerenciar e controlar o comportamento dos empregados (Carbonell \& Rodriguez-Escudero, 2013). A identidade corporativa atua como benefício intangível, principalmente quando a gestão adequada de recursos humanos torna a corporação mais atrativa para os empregados atuais e potenciais. O empregado com forte senso de identificação corporativa percebe seu trabalho de maneira mais positiva, o que leva à satisfação (Bravo, Matute, \& Pina, 2017).

Estudos anteriores relacionam que as avaliações positivas de identidade corporativa têm influência na satisfação, porque reputação corporativa sólida fornece ao indivíduo as recompensas psicológicas de associação com a corporação (Lin-Hi \& Blumberg, 2018; Podnar \& Golob, 2017). Portanto, torna-se possível formular a seguinte hipótese:

\section{Hipótese 3 (H3): A identidade corporativa impacta diretamente na satisfação do} empregado.

O diagrama de caminho mostrado na Figura 1 ilustra a relação causal, estabelecida pelas hipóteses, entre os construtos discutidos anteriormente. O diagrama de caminho utilizado teve como base o modelo conceitual de Barakat et al. (2016). Com substituição do construto imagem corporativa por identidade corporativa, buscando maior 
representatividade das relações hipotéticas e índices da modelagem de equações estruturais.

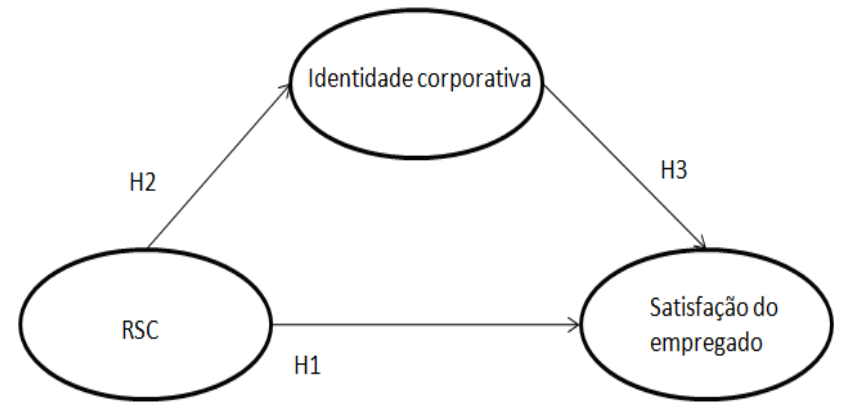

Figura 1: Diagrama de caminho do modelo hipotético.

Fonte: Adaptado de Barakat et al. (2016).

\section{MÉTODO}

Este estudo é de natureza explicativa, uma vez que tem a finalidade de avaliar nexos causais entre construtos (Gil, 2008).

\subsection{COLETA DE DADOS}

Realizou-se pesquisa de survey, contando com o questionário estruturado como instrumento de coleta de dados, meio pelo qual se procede à solicitação de informações ao grupo significativo de respondentes sobre o problema estudado. As conclusões sobre os dados coletados são obtidas mediante análise quantitativa. São extremamente úteis, pois proporcionam informações gerais acerca das populações, indispensáveis nas investigações das ciências sociais (Freitas, Oliveira, Saccol, \& Moscarola, 2000; Gil, 2008). Foi empregada escala Likert com cinco opções de resposta. De acordo com Hair Jr. et al. (2009), Likert é uma escala que tenta medir atitudes ou opiniões, cinco pontos são usados para avaliar a força da concordância ou discordância de uma pessoa com um conjunto de afirmações. Previamente à aplicação do questionário, realizou-se pesquisa qualitativa com 10 empregados de diferentes setores da corporação estudada, objetivando corrigir inconsistência que poderiam gerar ruído entre os respondentes, comprometendo a qualidade psicométrica do questionário.

No presente estudo, foram coletadas as opiniões dos empregados de uma corporação brasileira de capital aberto. Suas operações envolvem exploração e produção de petróleo, refino, vendas, transporte, petroquímica, distribuição de produtos derivados, gás natural e biocombustíveis. A corporação foi escolhida para o estudo porque 


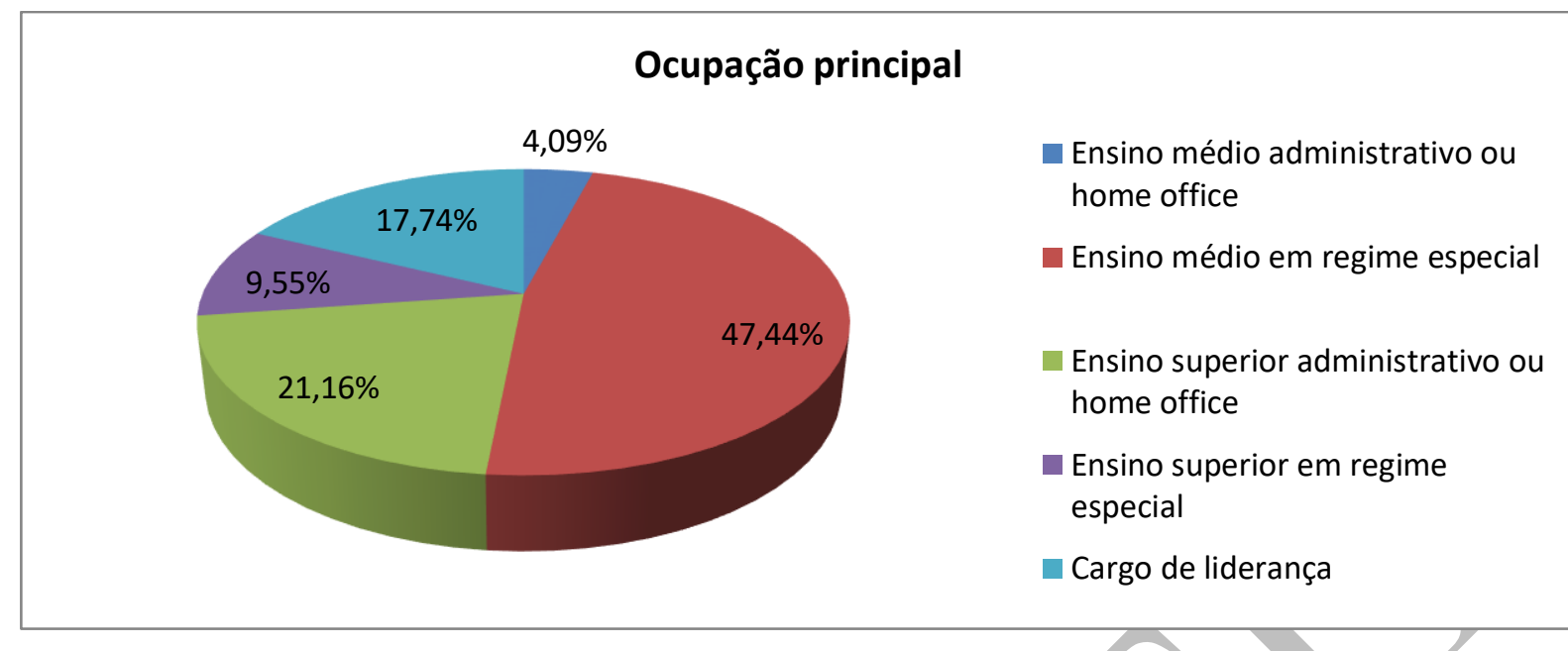

Figura 2: Ocupação principal dos respondentes.

Fonte: Elaboração própria

Na figura 2 é visualizada a proporção da ocupação principal na corporação estudada, fundamental para compreender a ausência de vieses na distribuição da relação de ganhos salariais dos empregados respondentes da pesquisa. A distinção de regime especial é orientada pelo recebimento de adicionais pelo trabalho conduzido em área industrial. Os maiores salários são compreendidos pelos empregados de nível superior e cargos de liderança (somando $48,47 \%$ dos empregados respondentes). No que tange ao nível de escolaridade dos respondentes, os dados representados na figura 3 revelam proporções distintas da população estudada.

\section{Escolaridade dos respondentes}

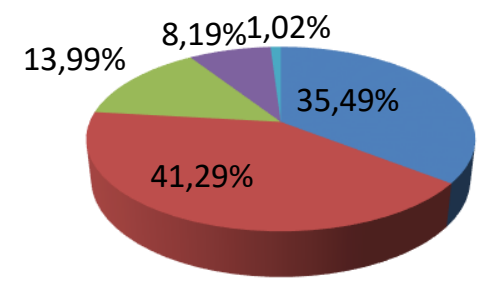
Ensino medio
- Graduação
Pós-graduação ou MBA
- Mestrado
Doutorado ou PhD

Figura 3: Escolaridade dos respondentes.

Fonte: Elaboração própria

\subsection{MODELO DE MENSURAÇÃO}

Em MEE com MQP, o modelo de mensuração, o qual também é referenciado como modelo externo, refere-se às relações das variáveis latentes com as variáveis observadas. A confiabilidade interna dos construtos foi verificada por intermédio dos 
indicadores Alfa de Cronbach e confiabilidade composta (CC). Ambos os indicadores verificam se a amostra possui vieses e se as variáveis observadas têm capacidade de gerar informações confiáveis (Hair Jr., Hult, Ringle, \& Sarstedt, 2016).

No que se refere ao coeficiente Alfa de Cronbach, espera-se encontrar valores superiores a 0,70, embora valores superiores a 0,60 sejam aceitáveis em pesquisas realizadas no campo das ciências sociais. No que tange à CC, espera-se encontrar valores entre 0,70 e 0,90, embora valores até 0,95 sejam aceitáveis (Hair Jr., Sarstedt, Hopkins, \& Kuppelwieser, 2014; Hair Jr. et al., 2016). Conforme pode ser observado na Tabela 1, os construtos do modelo desenvolvido para o presente estudo apresentaram confiabilidade interna adequada.

Tabela 1: Índices de Confiabilidade e Validade Convergente.

\begin{tabular}{|c|c|c|c|}
\hline Variável Latente & $\begin{array}{c}\text { Confiabilidade } \\
\text { Composta }\end{array}$ & $\begin{array}{c}\text { Alfa de } \\
\text { Cronbach }\end{array}$ & $\begin{array}{c}\text { Variância } \\
\text { Média Extraída }\end{array}$ \\
\hline $\begin{array}{c}\text { RSCORP } \\
\text { (Responsabilidade Social Corporativa) }\end{array}$ & 0,925 & 0,907 & 0,608 \\
\hline IDCORP & 0,883 & 0,801 & 0,715 \\
\hline (Identidade Corporativa) & 0,909 & 0,875 & 0,666 \\
\hline SATISF & & & 0 \\
\hline
\end{tabular}

Fonte: Elaboração própria.

Após a verificação da confiabilidade interna dos construtos, a análise do modelo de mensuração tem como segunda etapa, por meio do indicador variância média extraída (VME), a verificação da validade convergente dos construtos. Entende-se que valores de VME superiores a 0,50 são adequados, pois demonstra que o construto explica mais do que a média de variância de seus indicadores (Hair Jr. et al., 2016; Henseler, Hubona, \& Ray, 2016). Para tanto, foram estimadas as medidas das VME. Conforme pode ser visualizado na Tabela 1, os valores encontrados nos construtos foram satisfatórios.

Para avaliar a validade discriminante, deve ser considerado o critério de cargas cruzadas. Observa-se que as cargas das variáveis observadas participantes de cada construto são maiores no próprio construto, exibem magnitudes das cargas superiores a 0,708, apresentam significância estatística, assim como os sinais previstos pela teoria (Hair Jr. et al., 2014). Os valores encontrados no teste das cargas cruzadas podem ser avaliados na Tabela 2 . 
Tabela 2: Cargas e significância estatística dos indicadores do modelo.

\begin{tabular}{|l|c|c|c|c|c|c|}
\hline & RSCORP & IDCORP & SATISF & Tipo Modelo & Erro-padrão & Valor p \\
\hline Rscorp_1 & $\mathbf{0 , 7 5 2}$ & 0,075 & $-0,117$ & Refletivo & 0,052 & $<0,001$ \\
\hline Rscorp_2 & $\mathbf{0 , 7 2 1}$ & 0,077 & $-0,254$ & Refletivo & 0,052 & $<0,001$ \\
\hline Rscorp_3 & $\mathbf{0 , 8 1 4}$ & 0,034 & 0,060 & Refletivo & 0,051 & $<0,001$ \\
\hline Rscorp_4 & $\mathbf{0 , 8 1 5}$ & $-0,105$ & 0,012 & Refletivo & 0,051 & $<0,001$ \\
\hline Rscorp_5 & $\mathbf{0 , 7 9 1}$ & 0,004 & $-0,147$ & Refletivo & 0,052 & $<0,001$ \\
\hline Rscorp_6 & $\mathbf{0 , 8 4 3}$ & $-0,146$ & 0,002 & Refletivo & 0,051 & $<0,001$ \\
\hline Rscorp_7 & $\mathbf{0 , 7 5 2}$ & 0,052 & 0,153 & Refletivo & 0,052 & $<0,001$ \\
\hline Rscorp_8 & $\mathbf{0 , 7 3 9}$ & 0,036 & 0,286 & Refletivo & 0,052 & $<0,001$ \\
\hline Idcorp_1 & $-0,185$ & $\mathbf{0 , 8 1 1}$ & 0,390 & Refletivo & 0,051 & $<0,001$ \\
\hline Idcorp_2 & 0,168 & $\mathbf{0 , 8 5 5}$ & $-0,372$ & Refletivo & 0,051 & $<0,001$ \\
\hline Idcorp_3 & 0,008 & $\mathbf{0 , 8 7 0}$ & 0,002 & Refletivo & 0,051 & $<0,001$ \\
\hline Satisf_1 & $-0,042$ & $-0,215$ & $\mathbf{0 , 7 7 5}$ & Refletivo & 0,052 & $<0,001$ \\
\hline Satisf_2 & 0,274 & $-0,122$ & $\mathbf{0 , 8 3 2}$ & Refletivo & 0,051 & $<0,001$ \\
\hline Satisf_3 & $-0,178$ & $-0,059$ & $\mathbf{0 , 8 1 4}$ & Refletivo & 0,051 & $<0,001$ \\
\hline Satisf_4 & $-0,172$ & 0,106 & $\mathbf{0 , 8 3 1}$ & Refletivo & 0,051 & $<0,001$ \\
\hline Satisf_5 & 0,111 & 0,275 & $\mathbf{0 , 8 2 8}$ & Refletivo & 0,051 & $<0,001$ \\
\hline
\end{tabular}

Fonte: Elaboração própria.

A partir da análise dos dados da Tabela 2, torna-se possível identificar na variável latente exógena RSCORP os indicadores mais expressivos da pesquisa, justamente por apresentarem cargas mais elevadas dentro do construto. Rscorp_6 (bem-estar de pessoas idênticas aos empregados), Rscorp_4 (não discriminação na remuneração e promoção dos empregados) e Rscorp_3 (incentivo à diversidade da força de trabalho) foram os indicadores mais relevantes dentro das oito variáveis observadas do construto.

Com relação à variável latente IDCORP, o indicador mais relevante foi Idcorp_3 (orgulho percebido por empregados de outras corporações), seguido de Idcorp_2 (orgulho da comunidade) e Idcorp_1 (bom local de trabalho). Com relação à variável latente SATISF, os indicadores Satisf_2 (melhoria contínua no trabalho), Satisf_4 (estrutura de vida com o trabalho) e Satisf_5 (grupo de trabalho) apresentaram maior relevância no construto. Na Tabela 2, é possível observar que nenhum dos valores dos indicadores nos construtos aos quais pertencem foi menor que suas contribuições indiretas com quaisquer outros construtos do modelo. A próxima avaliação a ser executada é elementar a análise da validade discriminante do modelo, o critério de Fornell e Larcker (Hair Jr. et al., 2016). 
Tabela 3: Critério de Fornell e Larcker.

\begin{tabular}{|c|c|c|c|}
\hline \multicolumn{4}{|c|}{ Critério de Fornell e Larcker } \\
\hline & RSCORP & IDCORP & SATISF \\
\hline RSCORP & $\mathbf{0 , 7 7 9}$ & 0,696 & 0,766 \\
\hline IDCORP & 0,696 & $\mathbf{0 , 8 4 6}$ & 0,792 \\
\hline SATISF & 0,766 & 0,792 & $\mathbf{0 , 8 1 6}$ \\
\hline
\end{tabular}

Fonte: Elaboração própria.

A Tabela 3 apresenta a raiz quadrada da VME dos construtos do modelo. É possível verificar que os valores das raízes quadradas das VME de cada construto são maiores que as correlações entre outros construtos. Dessa forma, constata-se que o modelơ tem validade discriminante confirmada pelo critério de Fornell e Larcker, cuja abordagem é mais conservadora para assegurar a validade discriminante (Hair Jr. et al., 2016; Henseler, Hubona, \& Ray, 2016).

\subsection{MODELO ESTRUTURAL}

Previamente à análise do modelo estrutural, convém averiguar a existência de colinearidade entre as variáveis latentes, por intermédio da análise do fator de inflação da variância (mais comumente utilizado no idioma inglês - variance inflation factor - VIF). Conforme exibido na Tabela 4, é possível constatar que todas as variáveis apresentaram valores abaixo de 5, indicando a inexistência de problemas de colinearidade (Hair Jr. et al., 2009).

Tabela 4: Fator de inflação da variância do modelo.

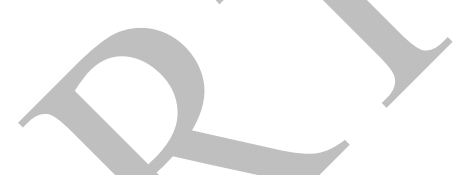

\begin{tabular}{|c|c|c|c|}
\hline \multicolumn{4}{|c|}{ Fator de inflação da Variância } \\
\hline & RSCORP & IDCORP & SATISF \\
\hline VIF & 2,553 & 2,828 & 3,526 \\
\hline
\end{tabular}

Fonte: Elaboração própria.

A conclusão da análise do modelo estrutural é determinada pela apresentação do diagrama de caminho do modelo hipotético, conforme pode ser verificado na Figura 4. 

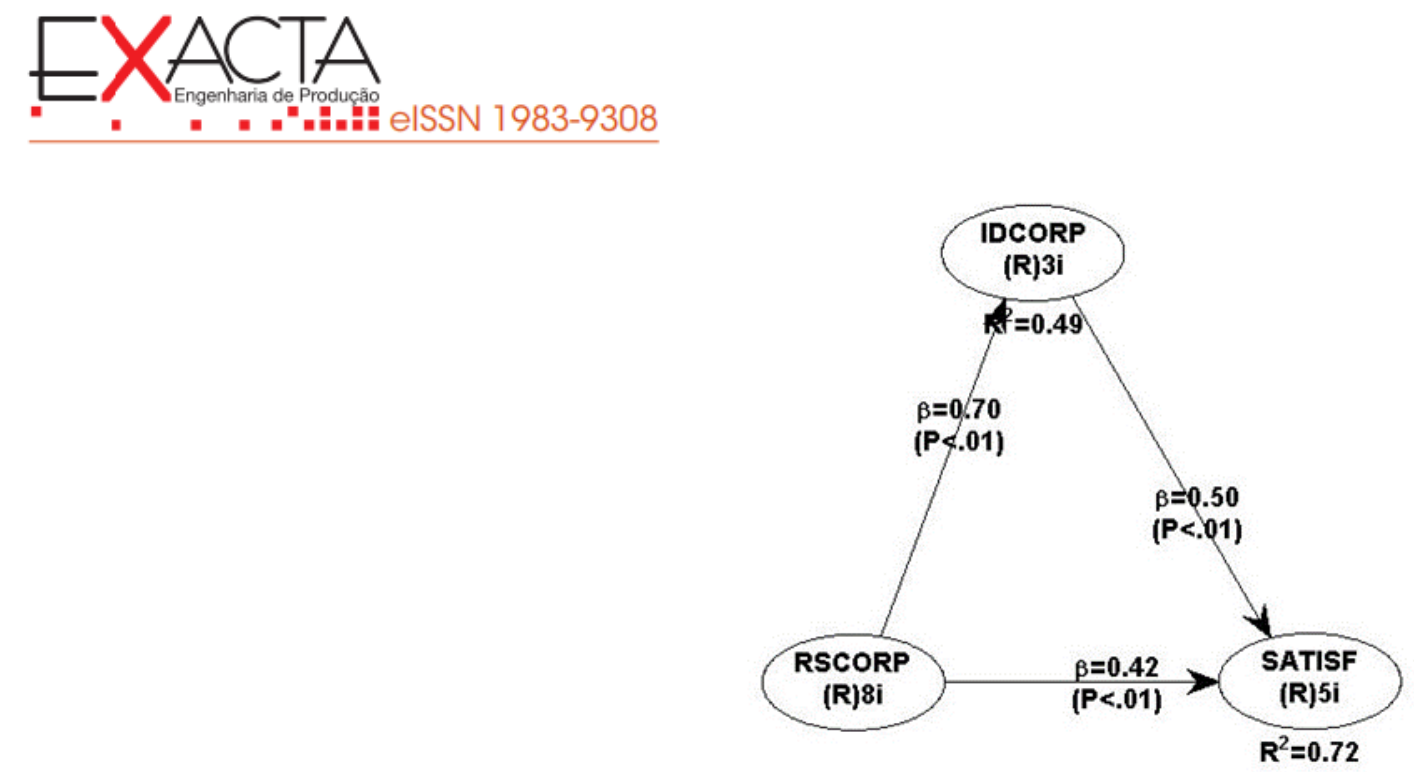

Figura 4: Diagrama de caminho e resultados do modelo hipotético.

Fonte: Elaboração própria.

A avaliação do modelo estrutural consistiu na apreciação do coeficiente de determinação de Pearson $\left(\mathrm{R}^{2}\right)$, que analisa os efeitos das variáveis latentes exógenas sobre as variáveis latentes endógenas. Os valores de $\mathrm{R}^{2}$ variam de 0 a 1 e quanto mais os valores se aproximam de 1, maior é a exatidão preditiva (Hair Jr. et al., 2016). Na presente pesquisa, constatou-se que o $\mathrm{R}^{2}$ alcançou para a variável latente endógena SATISF o valor de 0,72. Desse modo, o construto SATISF (satisfação do empregado) tem $72 \%$ de sua variação explicada pelo modelo estrutural. Averiguou-se ainda que $49 \%$ da variância da variável latente IDCORP (identidade corporativa) são explicados pelo modelo estrutural $\left(R^{2}=0,49\right)$. Os valores dos efeitos encontrados nos coeficientes $R^{2}$ são considerados bastante influentes na pesquisa em Ciências Sociais, valores superiores a 26\% expressam efeito relativamente grande à explicação de variância da variável latente endógena (Ringle, Da Silva, \& de Souza Bido, 2014).

No que se refere à avaliação da significância estatística dos coeficientes de caminho $(\beta)$, os resultados podem ser verificados na Tabela 5. O coeficiente $\beta$ representa o impacto da variável independente sobre a dependente. A condição de aceitabilidade é a existência de significância estatística; pode-se afirmar que representa as relações hipotéticas entre os construtos (Hair Jr. et al., 2014). Todas as relações hipotéticas entre as variáveis latentes do modelo apresentaram significância estatística a 1\%, confirmando todas as hipóteses apresentadas no presente estudo. 
Tabela 5: Valores dos coeficientes de caminho, nível de significância, tamanho do efeito e relação direta com as hipóteses da pesquisa.

\begin{tabular}{|c|c|c|c|c|}
\hline Caminho & $\boldsymbol{\beta}$ & $\mathbf{p}$ & $\mathbf{f}^{\mathbf{2}}$ & Relação direta \\
\hline $\mathrm{RSCORP} \rightarrow$ SATISF & 0,423 & $<0,001$ & 0,593 & Confirma Hipótese H1 \\
\hline $\mathrm{RSCORP} \rightarrow$ IDCORP & 0,700 & $<0,001$ & 0,490 & Confirma Hipótese H2 \\
\hline IDCORP $\rightarrow$ SATISF & 0,498 & $<0,001$ & 0,395 & Confirma Hipótese H3 \\
\hline
\end{tabular}

Fonte: Elaboração própria.

A Tabela 5 também apresenta os valores referentes aos índices de tamanho do efeito $\left(\mathrm{f}^{2}\right)$ ou indicadores de Cohen. Os valores $\mathrm{f}^{2}$ representam o nível de relevância do impacto de cada construto para o ajuste global do modelo. Todas as variáveis latentes exógenas apresentaram efeito forte (superiores de 0,35 ) em relação às variáveis latentes endógenas no modelo.

Com relação ao índice de relevância preditiva $\left(\mathrm{Q}^{2}\right)$ ou indicador de Stone-Geisser, os resultados do modelo estão apresentados na Tabela 6. Os valores para os construtos IDCORP e SATISF podem ser verificados com relevância preditiva grande (superiores de 0,35$)$, ou seja, o modelo se aproxima do que se espera dele.

Tabela 6: Índice de relevância preditiva do modelo.

Fonte: Elaboração própria.

\begin{tabular}{|c|c|}
\hline Variável latente & $\mathbf{Q}^{\mathbf{2}}$ \\
\hline IDCORP & 0,491 \\
\hline SATISF & 0,721 \\
\hline
\end{tabular}

\section{CONCLUSÕES}

O presente estudo exibiu evidência de que o modelo hipotético da pesquisa é adequado para prever a influência da RSC sobre a satisfação dos empregados, tendo como elemento mediador a identidade corporativa. Implicações adicionais vão de acordo com o trabalho de Brieger et al., (2020), que resultam do fato de que os efeitos da RSC na significância do trabalho e na identificação corporativa são mais fortes para empregados com maior consciência de valor público. Ou seja, indivíduos que mostram grande consideração pelo impacto de suas ações nas comunidades, na nação e no mundo como o todo.

Os resultados da pesquisa constataram que os empregados da corporação estudada valorizam as práticas e contribuições trazidas pela RSC para o seu local de trabalho. As 
relações dos construtos do modelo hipotético apresentaram efeitos de grande relevância, significância estatística e impacto positivo com todas as hipóteses confirmadas. Os resultados foram convergentes com o trabalho de Barakat et al., (2016), de fato, mostrando a existência de forte nexo causal entre a RSC e a satisfação dos empregados. A RSC explica $72 \%$ da variância da satisfação dos empregados. É um percentual muito expressivo. Os empregados são stakeholders cruciais para atingir as metas corporativas propostas e criar diferenciação na dinâmica de mercado.

O fato dos indicadores de RSC apresentarem as maiores cargas, está diretamente relacionado à prática da busca do bem-estar da comunidade. A corporação estudada divulgou em seu relatório de sustentabilidade, transparência das informações nas questões de governança corporativa (ESG), confirmando forte apreço na agenda social e práticas de compromisso de respeito aos direitos humanos, conforme os princípios do Pacto Global das Nações Unidas, inspirado nos Objetivos de Desenvolvimento Sustentável. No ano de 2019, os valores direcionados para doações totalizam R \$ 2,3 milhões.

O tratamento contra a discriminação na remuneração e promoção dos empregados está pautado na corporação estudada pela revisão anual de pesquisas de mercado, conduzidas por consultoria especializada, tais como Willis Towers Watson e Korn Ferry Hay Group. Apesar da predominante mão de obra masculina, existem programas internos de incentivo à diversidade da força de trabalho. Compromissos com os direitos humanos para o público interno são motivados por meio de Código de Ética e Conduta, combatendo assédios morais, raciais e sexuais na corporação.

Com relação à identidade corporativa nos resultados da pesquisa, foi possível perceber similaridade com o construto de Smidts et al., (2001), onde na visão dos empregados da corporação estudada, oferece reconhecimento e orgulho em relação a outras corporações do mesmo setor. Os indicadores da satisfação do trabalho mais relevantes, estão vinculados à postura de incentivo da melhoria contínua no trabalho e a melhora global da perspectiva de vida do empregado. Para alcançar essa contribuição positiva, torna-se necessário atribuir alguns fatores para as lideranças, como transmitir para as equipes o reconhecimento da competência técnica, do comprometimento, do companheirismo e do orgulho do que a corporação significa, enfatizando sua cultura.

A contribuição teórica deste estudo é a comprovação estatística da importância da contribuição direta da RSC na satisfação dos empregados. Pelos resultados obtidos nesta pesquisa, o modelo hipotético teve validade e forte impacto nas suas relações, poucos estudos brasileiros abordaram esta relação causal. Desta forma, o presente trabalho 
contribui para a literatura brasileira ao fornecer produção cientifica inerente à área do comportamento organizacional, a qual, por intermédio da técnica estatística de MEE com base em MQP, comprovou e explicou a influência positiva que a RSC respectivo a corporação brasileira exerce sobre a satisfação dos empregados, tendo a identidade corporativa como mediador.

Por fim, a contribuição prática desse estudo permite aos gestores das corporações melhor compreensão da influência exercida pela RSC, e como ampliar o nível de satisfação de seus empregados, possibilitando o aprimoramento de métodos de desenvolvimento do capital humano nas organizações. Exemplos como a promoção da meritocracia, o foco em resultados, a atuação integrada, a inovação e a superação de desafios, gerando conseqüentes vantagens competitivas para as corporações ho mercado.

\subsection{RECOMENDAÇÕES PARA FUTURAS PESQUISAS}

Com objetivo de tornar o modelo cada vez mais confiável, recomenda-se utilizá-lo com empregados de outros setores. O modelo hipotético teve seu foco voltado para corporação do segmento industrial. Estudos adicionais podem contemplar outros segmentos econômicos, incluindo também corporações da esfera de serviços e comércio.

Recomenda-se também a utilização do modelo por meio da técnica estatística de modelagem de equações estruturais, com base em matriz de covariância. Por fim, recomenda-se que o modelo também seja utilizado em outros países e economias, visando verificar se o resultado encontrado varia de acordo com o tipo de contexto corporativo que for submetido.

\section{REFERÊNCIAS BIBLIOGRÁFICAS}

Abratt, R., \& Mingione, M. (2017). Corporate identity, strategy and change. Journal of Brand Management, 24(2), 129-139. https://doi.org/10.1057/s41262-017-0026-8.

Aguilera, R. V., Rupp, D. E., Williams, C. A., \& Ganapathi, J. (2007) Putting the S back in corporate social responsibility: a multilevel theory of social change in organizations. $\begin{array}{lllll}\text { Academy of } & \text { Management } & \text { 836-863. }\end{array}$ https://doi.org/10.5465/amr.2007.25275678.

Allameh, S. M. (2018). Antecedents and consequences of intellectual capital. Journal of Intellectual Capital, 19 (5), 858-874. https://doi.org/10.1108/JIC-05-2017-0068. 
Ashton, A. S. (2018). How human resources management best practice influence employee satisfaction and job retention in the Thai hotel industry. Journal of Human

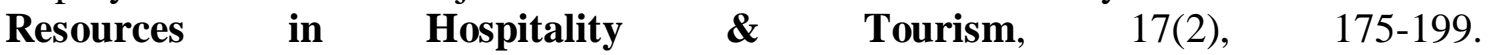
https://doi.org/10.1080/15332845.2017.1340759.

Balmer, J. M. (2017). The corporate identity, total corporate communications, stakeholders' attributed identities, identifications and behaviours continuum. European Journal of marketing. 51(9/10), 1472-1502. https://doi.org/10.1108/EJM-07-20170448 .

Barakat, S. R., Isabella, G., Boaventura, J. M. G., \& Mazzon, J. A. (2016) The influence of corporate social responsibility on employee satisfaction. Management decision, 54(9), 2325-2339. https://doi.org/10.1108/MD-05-2016-0308.

Bartels, J., Pruyn, Ad, De Jong, M., \& Joustra, I. (2007) Multiple organizational identification levels and the impact of perceived external prestige and communication climate. Journal of Organizational Behavior: The International Journal of Industrial, Occupational and Organizational Psychology and Behavior, 28(2), 173-190. https://doi.org/10.1002/job.420.

Boğan, E., \& Dedeoğlu, B. B. (2020). Hotel employees' corporate social responsibility perception and organizational citizenship behavior: Perceived external prestige and pride in organization as serial mediators. Corporate Social Responsibility and Environmental Management, 27(5), 2342-2353. https://doi.org/10.1002/csr.1996.

Book, L., Gatling, A., \& Kim, J. (2019). The effects of leadership satisfaction on employee engagement, loyalty, and retention in the hospitality industry. Journal of Human Resources in Hospitality \& Tourism, 18(3), 368-393. https://doi.org/10.1080/15332845.2019.1599787.

Bowen, H. R. (1953) Social Responsibilities of the Businessman. New York: Harper \& Row.

Bravo, R., Matute, J., \& Pina, J. M. (2017). Corporate identity management and employees' responses. Journal of Strategic Marketing, 25(1), 1-13. https://doi.org/10.1080/0965254X.2015.1076876.

Brieger, S. A., Anderer, S., Fröhlich, A., Bäro, A., \& Meynhardt, T. (2020). Too much of a good thing? On the relationship between CSR and employee work addiction. Journal of Business Ethics, 166(2), 311-329. https://doi.org/10.1007/s10551-019-04141-8.

Brundtland, G. H. (1987) Our common future: call for action. Environmental Conservation, 14(4), 291-294. https://www.jstor.org/stable/44518052.

Caldwell, C., \& Peters, R. (2018). New employee onboarding-psychological contracts and ethical perspectives. Journal of Management Development, 37(1), 27-39. https://doi.org/10.1108/JMD-10-2016-0202.

Carbonell, P. \& Rodriguez-Escudero, A. I. (2013) Management control, role expectations and job satisfaction of new product development teams: the moderating effect of 
participative decision-making. Industrial Marketing Management, 42(2), 248-259. https://doi.org/10.1016/j.indmarman.2012.08.007.

Carroll, A. B. (1979) A three-dimensional conceptual model of corporate performance. $\begin{array}{lllll}\text { Academy of } & \text { Management } & \text { 4(4), } & \text { 497-505. }\end{array}$ https://doi.org/10.5465/amr.1979.4498296.

Chang, C-H. (2015) Proactive and reactive corporate social responsibility: antecedent and consequence. Management Decision, 53(2), 451-468. https://doi.org/10.1108/MD-022014-0060.

Dhanesh, G. S. (2014). CSR as organization-employee relationship management strategy: A case study of socially responsible information technology companies in India. $\begin{array}{lllll}\text { Management } & \text { Communication } & \text { Quarterly, } & \text { 28(1), } & \text { 130-149. }\end{array}$ https://doi.org/10.1177/0893318913517238.

Dutton, J. E., \& Dukerich, J. M. (1991) Keeping an eye on the mirror: image and identity in organizational adaptation. Academy of Management Journal, 34(3), 517-554. https://doi.org/10.5465/256405.

Eells, R. S. F., \& Walton, C. C. (1969) Conceptual Foundations of Business. Homewood, IL: RD Irwin.

El Akremi, A., Gond, J-P., Swaen, V., De Roeck, K., \& Igalens, J. (2018) How do employees perceive corporate responsibility? Deyelopment and validation of a multidimensional corporate stakeholder responsibility scale. Journal of Management, 44(2), 619-657. https://doi.org/10.1177/0149206315569311.

El-Kassar, A. N., Yunis, M., \& El-Khalil, R. (2017). The mediating effects of employeecompany identification on the relationship between ethics, corporate social responsibility, and organizational citizenship behavior. Journal of Promotion Management, 23(3), 419-436. https://doi.org/10.1080/10496491.2017.1294872.

Freitas, H., Oliveira, M., Saccol, A. Z., \& Moscarola, J. (2000). O método de pesquisa survey. Revista de Administração da Universidade de São Paulo - RAUSP, 35(3), 105-112.

Gil, A. C. (2008) Métodos e técnicas de pesquisa social. 6. ed. Editora Atlas SA.

Hair, J. F., Black, W. C., Babin, B. J., Anderson, R. E., \& Tatham, R. L. (2009). Análise multivariada de dados. Bookman editora.

Hair Jr., J. F., Sarstedt, M., Hopkins, L., \& Kuppelwieser, V. G. (2014) Partial least squares structural equation modeling (PLS-SEM): an emerging tool in business research, European Business Review, 26(2), 106-121. https://doi.org/10.1108/EBR-10-20130128.

Hair Jr., J. F., Hult, T. M., Ringle, C. M., \& Sarstedt, M. (2016) A Primer on Partial Least Squares Structural Equation Modeling (PLS-SEM). Los Angeles: SAGE. 


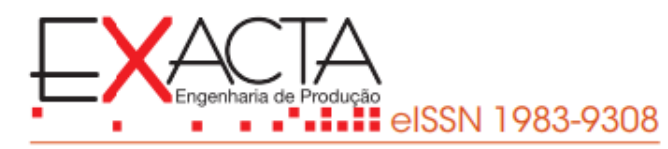

Hair Jr., J. F., \& Sarstedt, M. (2019) Factors versus composites: guidelines for choosing the right structural equation modeling method. Project Management Journal, 50(6), 619-624. https://doi.org/10.1177/8756972819882132.

Harter, J. K., Schmidt, F. L., \& Hayes, T. L. (2002) Business-unit-level relationship between employee satisfaction, employee engagement, and business outcomes: a metaanalysis. Journal of Applied Psychology, 87(2), 268. https://doi.org/10.1037//00219010.87.2.268.

Henseler, J., Hubona, G., \& Ray, P. A. (2016) Using PLS path modeling in new technology research: updated guidelines. Industrial Management \& Data Systems, 116(1), 2-20. https://doi.org/10.1108/IMDS-09-2015-0382.

Hoang, T. (2018) The role of the integrated reporting in raising awareness of environmental, social and corporate governance (ESG) performance. In: Stakeholders, governance and responsibility (Developments in Corporate Governance and Responsibility). Emerald Publishing Limited, 14, 47-69. https://doi.org/10.1108/S2043052320180000014003.

Haski-Leventhal, D., Roza, L., \& Meijs, L. C. (2017). Congruence in corporate social responsibility: Connecting the identity and behavior of employers and employees. Journal of Business Ethics, 143(1), 35-51. https://doi.org/10.1007/s10551-015-2793-z.

Hon, L. C., \& Grunig, J. E. (1999) Guidelines for measuring relationships in public relations. Gainesville, FL: Institute for Public Relations.

Judge, T. A., Weiss, H. M., Kammeyer-Mueller, J. D., \& Hulin, C. L. (2017). Job attitudes, job satisfaction, and job affect: A century of continuity and of change. Journal of Applied Psychology, 102(3), 356. https://doi.org/10.1037/ap10000181.

Jung, H. S., \& Yoon, H. H. (2015) The impact of employees' positive psychological capital on job satisfaction and organizational citizenship behaviors in the hotel. International Journal of Contemporary Hospitality Management, 27(6), 1135-1156. https://doi.org/10.1108/IJCHM-01-2014-0019.

Kampkötter, P. (2017). Performance appraisals and job satisfaction. The International Journal of Human Resource Management, 28(5), 750-774. https://doi.org/10.1080/09585192.2015.1109538.

Khaskheli, A., Jiang, Y., Raza, S. A., Qureshi, M. A., Khan, K. A., \& Salam, J. (2020). Do CSR activities increase organizational citizenship behavior among employees? Mediating role of affective commitment and job satisfaction. Corporate Social Responsibility and Environmental Management, 27(6), 2941-2955. https://doi.org/10.1002/csr.2013.

Krémer, V. (2019). The impact of CSR on job satisfaction and employee retention in Hungarian hotels. In International dimensions of sustainable management (pp. 307327). Springer, Cham. https://doi.org/10.1007/978-3-030-04819-8_19.

Lee, J., \& Lee, Y. (2018). Effects of multi-brand company's CSR activities on purchase intention through a mediating role of corporate image and brand image. Journal of 
Fashion Marketing and Management: An International Journal, 22(3), 387-403. https://doi.org/10.1108/JFMM-08-2017-0087.

Lee, Y. (2020) Toward a communality with employees: the role of CSR types and internal $\begin{array}{lllll}\text { reputation. Corporate } & \text { Reputation } & \text { Review, 23(1), }\end{array}$ https://doi.org/10.1057/s41299-019-00069-x.

Lin-Hi, N., \& Blumberg, I. (2018). The link between (not) practicing CSR and corporate reputation: Psychological foundations and managerial implications. Journal of Business ethics, 150(1), 185-198. https://doi.org/10.1007/s10551-016-3164-0.

Locke, E. A. (1976) The nature and causes of job satisfaction: handbook of industrial and organizational psychology. Chicago: RandMcNarlly.

McCarthy, L. (2018). "There is no time for rest": Gendered CSR, sustainable development and the unpaid care work governance gap. Business Ethics: A European Review, 27(4), 337-349. https://doi.org/10.1111/beer.12190.

Murmura, F., Bravi, L., \& Palazzi, F. (2017) Evaluating companies' commitment to corporate social responsibility: perceptions of the SA 8000 standard. Journal of Cleaner Production, 164, 1406-1418. https://doi.org/10.1016/j.jclepro.2017.07.073.

Murshed, F., Sen, S., Savitskie, K., \& Xu, H. (2021). CSR and job satisfaction: Role of CSR importance to employee and procedural justice. Journal of Marketing Theory and Practice, 1-16. https://doi.org/10.1080/10696679.2021.1877156.

Odriozola, M. D., \& Baraibar-Diez, E. (2017) Is corporate reputation associated with quality of CSR reporting? Evidence from Spain. Corporate Social Responsibility and Environmental Management, 24(2), 121-132. https://doi.org/10.1002/csr.1399.

Onkila, T. (2015) Pride or embarrassment? Employees' emotions and corporate social responsibility. Corporate Social Responsibility and Environmental Management, 22(4), 222-236. https://doi.org/10.1002/csr.1340.

Pinelli, M., \& Maiolini, R. (2017). Strategies for sustainable development: Organizational motivations, stakeholders' expectations and sustainability agendas. Sustainable Development, 25(4), 288-298. https://doi.org/10.1002/sd.1653.

Podnar, K., \& Golob, U. (2017). The quest for the corporate reputation definition: Lessons from the interconnection model of identity, image, and reputation. Corporate Reputation Review, 20(3), 186-192. https://doi.org/10.1057/s41299-017-0027-2.

Ramesh, K., Saha, R., Goswami, S., \& Dahiya, R. (2019). Consumer's response to CSR activities: Mediating role of brand image and brand attitude. Corporate Social Responsibility and Environmental Management, 26(2), 377-387. https://doi.org/10.1002/csr.1689.

Ringle, C. M., Da Silva, D., \& de Souza Bido, D. (2014) Modelagem de equações estruturais com utilização do SmartPLS. Revista Brasileira de Marketing, 13(2), 56-73. DOI: $10.5585 /$ remark.v13i2.2717. 
